\title{
Assessing and improving human movements using sensitivity analysis and digital human simulation
}

\author{
Pauline Maurice $^{\mathrm{a}, \mathrm{b}, \mathrm{d}}$, Vincent Padois ${ }^{\mathrm{b}, \mathrm{c}}$, Yvan Measson $^{\mathrm{d}}$ and Philippe Bidaud ${ }^{\mathrm{b}, \mathrm{e}}$ \\ ${ }^{a}$ Université de Lorraine, CNRS, Inria, LORIA, F-54000 Nancy, France; borbonne \\ Université, CNRS UMR 7222, Institut des Systèmes Intelligents et de Robotique, ISIR,

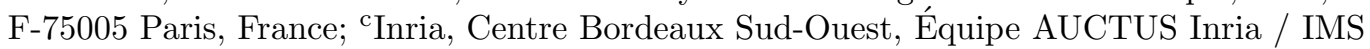 \\ (Univ. Bordeaux, CNRS UMR5218), F-33405 Talence, France; ${ }^{\mathrm{d}}$ CEA, LIST, Interactive \\ Robotics Laboratory, Gif-sur-Yvette, F-91191, France; ${ }^{e}$ ONERA, 91123 Palaiseau, France
}

\begin{abstract}
Enhancing the performance of technical movements aims both at improving operational results and at reducing biomechanical demands. Advances in human biomechanics and modeling tools allow to evaluate human performance with more and more details. Finding the right modifications to improve the performance is, however, still addressed with extensive time consuming trial-and-error processes. This paper presents a framework for easily assessing human movements and automatically providing recommendations to improve their performances. An optimization-based whole-body controller is used to dynamically replay human movements from motion capture data, to evaluate existing movements. Automatic digital human simulations are then run to estimate performance indicators when the movement is performed in many different ways. Sensitivity indices are thereby computed to quantify the influence of postural parameters on the performance. Based on the results of the sensitivity analysis, recommendations for posture improvement are provided. The method is successfully validated on a drilling activity.
\end{abstract}

\section{KEYWORDS}

Digital human simulation, Dynamic motion replay, Sensitivity analysis of human motion, Ergonomics.

\section{Introduction}

Performance enhancement in technical postures or movements has always been of great concern. In numerous applications the overall performance is twofold, consisting of both the achievement of some operational goal and the minimization of the biomechanical demands experienced by the person. Workstations designers now often take into account the exposure to musculoskeletal disorders risk factors in addition to workers' productivity (Schneider and Irastorza 2010; NRC 2001). In sports, coaches aim at finding the right movement to improve athletes' results while preventing injuries (Fortenbaugh, Fleisig, and Andrews 2009; Robinson and ODonoghue 2008). In rehabilitation, knowing which motion patterns alleviate the stress on a weakened body part helps provide exercises or recommendations to prevent further injury (Sturnieks et al. 2008).

The assessment and improvement of a movement are usually conducted under the supervision of an expert (e.g. ergonomist, physiotherapist) who observes the person 
performing the activity and provides recommendations based on his/her knowledge and experience. The availability of experts may however be limited. Besides, observational methods provide only qualitative measures of the biomechanical demands experienced by the person. Digital human software (software in which human motions are reproduced with a digital human model) have therefore been developed to assist and supplement experts (e.g. OpenSim (Delp et al. 2007), AnyBody (Damsgaard et al. 2006)). Such software enable easy access to detailed force and motion-related biomechanical quantities, which otherwise can only be measured on real humans with complex instrumentation, if at all (e.g. muscle or joint forces). However, the reliability of these biomechanical measurements is questionable (Hicks et al. 2015). One key factor is the mapping of the human motion onto the digital model: the resulting motion should be dynamically consistent (i.e. respect the laws of physics) to enhance the reliability of force-related quantities. Yet, existing software do not guarantee such consistency (Hicks et al. 2015).

The other difficulty in improving technical movements lies in the identification of suitable modifications which will enhance the performance (Fig. 1). Relations between the macroscopic parameters of the movement (i.e. adjustable parameters defining the way the movement is performed) and the resulting performances are often complex. Therefore, despite advances in human biomechanics and modeling tools, successful modifications generally still result from an intensive trial-and-error process. In (Demircan 2012), Demircan proposes a tool for analyzing the relation between novice and expert athletes' movements and the resulting performances. This tool reveals the features differentiating an efficient movement from a non-efficient one, but cannot provide explicit recommendations for an optimal execution. Besides, a single performance criterion is considered (tool acceleration), whereas a detailed assessment of biomechanical performances relies on several quantities which may be differently affected by a same parameter of the movement (e.g. joint loads, joint positions, energy consumption).

This paper presents a framework to assess the performance of a technical movement and easily identify how to improve it, while addressing the aforementioned concerns. The proposed framework consists of two components:

- A method for replaying pre-recorded human motions while ensuring the dynamic consistency of the resulting motion. This dynamic replay relies on an optimization-based controller which enables to track subjects' motion in operational space, while imposing dynamic and biomechanical constraints. Existing situations can thus be evaluated.

- A method for analyzing the dependence between parameters and performances of the movement. A variety of situations are automatically created and evaluated using an autonomous digital human model (no motion capture), and a sensitivity analysis is conducted on the simulation results. The critical parameters of the movement can thus be identified and tuned, using only little input data.

The paper is organized as follows. Section 2 presents the two components of the framework, with an emphasis on the dynamic replay method. The sensitivity analysis part has already been published in (Maurice et al. 2017) and is only briefly described here. Section 3 describes the experimental set-up which is used as a proof of concept of the proposed framework. The results are presented in section 4 and discussed in section 5 . 


\section{Method}

Human motion is often captured through optical motion capture techniques, in which markers are positioned on the human body and cameras record the markers 3D positions. The recorded markers trajectories are then mapped onto a digital human model (DHM) to estimate movement-related biomechanical quantities. Despite their extensive use, the current mapping techniques still lack physical consistency, especially when the motion is highly dynamic and/or involves significant interaction forces with the environment. Note that the retargetting problem (i.e. mapping the motion of a subject onto an avatar with a different morphology is not considered here. This work assumes that the DHM morphology can be adapted to each subject, and that its kinematics is very similar to the human body kinematics.

\subsection{Dynamic replay of human motion: Related work}

Recorded human motions are commonly mapped onto a DHM with inverse kinematics techniques (IK), which convert markers operational (i.e. Cartesian) space trajectories into joint space trajectories. With IK, kinematic quantities such as joint positions and velocities can be measured. Conversely, the estimation of the driving forces (joint torques or muscle forces) requires an additional inverse dynamics (ID) step, in which forces are computed from the DHM dynamic model and the joint space trajectories resulting from IK. Though widely used, the IK+ID process has several drawbacks. First, the IK step can be time consuming. Second, the IK solution is not unique, so the resulting motion may not be plausible. Many authors address this concern by using a modified IK to match the resulting motion with a given set of constraints (Lee and Shin 1999; Grochow et al. 2004). However, the dynamic properties of the human body are not considered so the computed motion is not dynamically consistent (such techniques are especially used in computer animation, where the visual realism is the primary concern). This inconsistency prevents the force equilibrium in the ID step when experimental external forces are added (e.g. ground reaction force). In OpenSim, for instance, this inconsistency appears in residual forces (Hicks et al. 2015).

In order to improve the dynamic consistency of the replayed motion, some studies include dynamic considerations in the motion computation. Multon et al. (Multon et al. 2009) combine IK with dynamic corrections, modifying motion capture data to respond to physical collision forces. Da Silva et al. (Da Silva, Abe, and Popović 2008) and Muico et al. (Muico et al. 2009) directly use controller-based techniques including dynamic constraints to animate a DHM, but they still require the joint trajectories resulting from IK as an input.

To avoid the IK step, some authors work directly in the operational space. John and Dariush (John and Dariush 2014) use a task space kinematic control method (closedloop inverse kinematics) and dynamic constraints to track the motion directly in task space. Ott et al. (Ott, Lee, and Nakamura 2008) connect the markers to the DHM body with virtual springs, and use the generated forces to compute the DHM motion through the dynamic model equation. Demircan et al. (Demircan et al. 2010) use an operational space approach based on null-space projection (Khatib 1987) to track the Cartesian markers trajectories. However, these techniques cannot explicitly take into account certain constraints of the movement. Specifically, inequality constraints such as joint limits cannot be included in null-space projection techniques. Instead, authors resort to suboptimal heuristics to account for inequality constraints through avoidance 
tasks (Sentis and Khatib 2006), or simply dismiss these constraints.

\subsection{DHM controller}

The dynamic replay method presented in this work is based on a direct control of markers in the operational space through an optimization-based controller. Unlike analytical techniques such as explicit null-space projection (Khatib 1987), numerical optimization-based techniques enable to solve the human kinematic redundancy while explicitly taking into account both equality and inequality constraints.

The DHM used here is a rigid body model which does not include muscles. Each joint is controlled by a single ideal rotational actuator, so the actuation variables are the joint torques. Though muscle-related quantities cannot be measured with such a model, biomechanical demands can be estimated with quantities such as joint loads, joint dynamics, or mechanical energy. Besides, while musculoskeletal models have proved valid and insightful in specific cases, no general criterion has been established yet to solve the muscle recruitment problem. This is a concern for the reliability of muscle-related measurements (Hicks et al. 2015; Thelen, Anderson, and Delp 2003; Damsgaard et al. 2006; Chaffin, Andersson, and Martin 2006). The questionable gain of information and the significantly higher computational cost therefore reduces the interest of musculoskeletal models in the current context.

The DHM motion is computed with the linear quadratic programming (LQP) controller framework developed by Salini et al. (Salini, Padois, and Bidaud 2011). LQP handles the optimization of a quadratic objective that depends on variables subjected to linear equality and inequality constraints. The variables are the joint torques, but also the contact forces. The ground reaction force (GRF) is therefore computed in the optimization process, and does not need to be recorded beforehand to replay the motion. The GRF estimation is a significant advantage over most of the other motion replay techniques since it simplifies the experimental set-up. The control problem is formulated as follows:

$$
\begin{array}{ll}
\underset{\mathbb{X}}{\operatorname{argmin}} \sum_{i} \omega_{i} T_{i}(\mathbb{X}) \\
\text { s.t. }\left\{\begin{array}{l}
M(\mathbf{q}) \dot{\boldsymbol{\nu}}+\mathbf{C}(\mathbf{q}, \boldsymbol{\nu})+\mathbf{g}(\mathbf{q})=S \boldsymbol{\tau}-\sum_{j} J_{c_{j}}^{T}(\mathbf{q}) \mathbf{w}_{\mathbf{c}_{\mathbf{j}}} \\
G \mathbb{X} \preceq \mathbf{h}
\end{array}\right.
\end{array}
$$

where $\boldsymbol{\tau}$ is the vector of joint torques, $\mathbf{w}_{\mathbf{c}_{\mathbf{j}}}$ the contact wrench of the $j$-th contact point, $\mathbf{q}$ the generalized coordinates of the system (i.e. joint angles), $\boldsymbol{\nu}$ the generalized velocity concatenating the free-floating base twist and the joint velocities $\dot{\mathbf{q}}$, and $\mathbb{X}=$ $\left(\boldsymbol{\tau}^{T}, \mathbf{w}_{\mathbf{c}}{ }^{T}\right)^{T}$. The equality constraint is the equation of motion; $M$ is the inertia matrix of the system, $\mathbf{C}$ the vector of centrifugal and Coriolis forces, $\mathbf{g}$ the vector of gravity forces, $S$ the actuation selection matrix due to the free-floating base, and $J_{c}^{T}$ the Jacobian of contacts. The inequality constraint includes the bounds on joint positions, velocities, and torques formulated in $\tau$, and the contact existence conditions for each contact point according to the Coulomb friction model:

$$
\begin{aligned}
& C_{c_{j}} \mathbf{w}_{\mathbf{c}_{\mathbf{j}}} \leq 0 \quad \forall j \\
& J_{c_{j}}(\mathbf{q}) \dot{\boldsymbol{\nu}}+\dot{J}_{c_{j}}(\boldsymbol{\nu}, \mathbf{q}) \boldsymbol{\nu}=0 \quad \forall j
\end{aligned}
$$


where $C_{c_{j}}$ is the linearized friction cone of the $j$-th contact point.

The objective function is a weighted sum (weights $\omega_{i}$ ) of tasks $T_{i}$ representing the squared error between a desired acceleration or wrench and the system acceleration/wrench. The solution is a compromise between the different tasks, based on their relative weights (the proposed method could however easily be adapted to a strict priority strategy such as hierarchical quadratic programming (Escande, Mansard, and Wieber 2014)). Four categories of tasks are defined by the following errors:

- Operational space acceleration

$$
\begin{aligned}
& \left\|J_{i} \dot{\boldsymbol{\nu}}+\dot{J}_{i} \boldsymbol{\nu}-\ddot{\mathbf{X}}_{\mathbf{i}}^{*}\right\|^{2} \\
& \left\|\ddot{\mathbf{q}}-\ddot{\mathbf{q}}^{*}\right\|^{2} \\
& \left\|\mathbf{w}_{\mathbf{i}}-\mathbf{w}_{\mathbf{i}}^{*}\right\|^{2} \\
& \left\|\boldsymbol{\tau}-\boldsymbol{\tau}^{*}\right\|^{2}
\end{aligned}
$$$$
\text { - Joint space acceleration }
$$$$
\text { - Operational space wrench }
$$$$
\text { - Joint torque }
$$

where $\ddot{\mathbf{X}}_{\mathbf{i}}$ is the Cartesian acceleration of body $i$, and $\mathbf{w}_{\mathbf{i}}$ the wrench associated with body $i$. The superscript * refers to the desired acceleration/wrench/torque. The desired acceleration is defined by a proportional derivative control:

$$
\ddot{\mathbf{z}}^{*}=\ddot{\mathbf{z}}^{\text {goal }}+K_{v}\left(\dot{\mathbf{z}}^{\text {goal }}-\dot{\mathbf{z}}\right)+K_{p}\left(\mathbf{z}^{\text {goal }}-\mathbf{z}\right)
$$

where $\mathbf{z}$ stands for $\mathbf{X}$ or $\mathbf{q}$, and $K_{p}$ and $K_{v}$ are the proportional and derivative gains. The superscript goal indicates the position, velocity and acceleration wanted for the body or joint (reference trajectory). Note that the acceleration variable $\dot{\boldsymbol{\nu}}$ can be expressed as a function of $\mathbb{X}$ using the equation of motion.

\subsection{Tasks for motion replay}

The recorded motion is mapped onto the DHM by creating an operational acceleration task for each marker placed on the subject's body, and using the recorded marker trajectories as reference trajectories (Fig. 2). Due to unavoidable differences between the human and DHM kinematics, the markers tracking tasks alone are often not sufficient to maintain the DHM balance (the balance is in open-loop control and the DHM falls). A center of mass (CoM) acceleration task is therefore added to control balance. The reference CoM acceleration is computed with a Zero Moment Point (ZMP) preview control method (Kajita et al. 2003). Additionally, if the activity includes exerting intentional force on the environment (e.g. pushing an object), an operational space wrench task is created at the hand. The original ZMP preview control scheme is modified to take into account these known external forces acting on the DHM. Unlike the GRF, the intentional reference force must be given as an input to the controller (e.g. from force sensor measurement or known object features). In order to obtain a natural posture even when some body segments are not entirely constrained by the markers tracking tasks, preferred joint angles are specified with joint acceleration tasks (corresponding to a standing posture arms along the body). Eventually, joint torque minimization tasks are added to prevent useless effort and ensure the uniqueness of the solution to the optimization problem.

Given the high number of tasks in the controller and the differences between the human and DHM kinematics, not all tasks can be fully fulfilled. The weighting strategy of the controller allows to deal with conflicting objectives, but tasks weights nevertheless affect the resulting motion. The balance task, for instance, is required to prevent the 

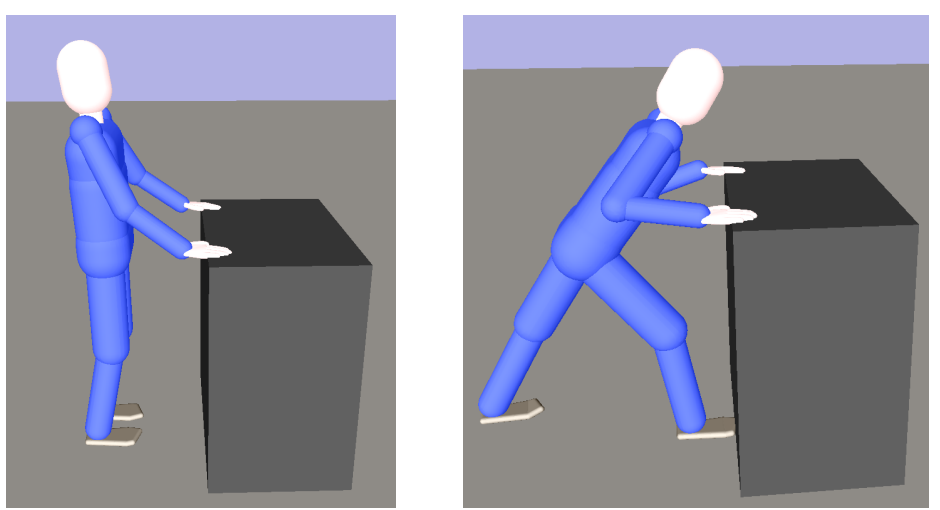

Figure 1. Two different ways of pushing a heavy object, resulting in different biomechanical demands on the human body (inspired from (Demircan 2012)).

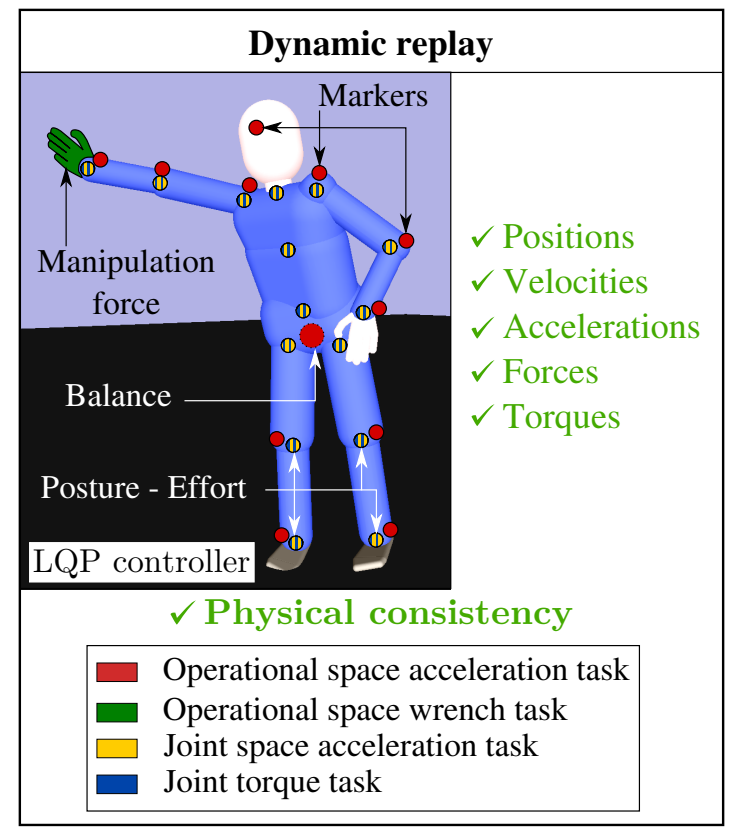

Figure 2. Joint space and operational space tasks used in the LQP controller for the dynamic replay of human motion. 
DHM from falling, but alters the lower and mid-body markers tracking tasks. Approximate values of tasks weights are first determined from common sense. In accordance with Demircan et al. (Demircan et al. 2010), distal markers tasks are assigned larger weights than proximal markers tasks to reduce the effect of cumulative errors in preceding joint positions. Joint space tasks (torque minimization and preferred posture) have the lowest weights since they are background tasks. Weights are then manually tuned by trial-and-error. Though time consuming in the first place, the tuning process does not need to be repeated; the weights obtained are general enough to be used for successfully replaying many different activities. The weights values are given in Table 1.

\subsection{Sensitivity analysis of human performances}

The dynamic replay method allows easy measurement of operational and biomechanical performances with a DHM. But these measurements alone do not give information about the postural changes which would enhance the overall performance. Providing postural recommendations requires to know the influence of the adjustable postural parameters on the movement performance. Most of the time, however, no straightforward analytical relation between parameters and performances is available. This work therefore proposes to establish parameter-performance influence through a statistical sensitivity analysis. The sensitivity analysis method has already been published in (Maurice et al. 2017) in the context of collaborative robots assessment. The method is summarized below, with a focus on its application to movement improvement.

Statistical sensitivity analysis relies on numerical evaluation of the output (indicators of performance) for numerous values of the input parameters (Saltelli, Chan, and Scott 2000). Given the large number of trials required, movements are simulated with an autonomous DHM so that many situations can rapidly be tested without the need for any human subjects. Unlike motion capture and replay, the motion of an autonomous DHM is automatically generated from high-level descriptions of the tasks to execute. The whole process for analyzing the dependence between the postural parameters of a given movement and the resulting performance is summarized as follows (Fig. 3):

(1) Define the adjustable parameters characterizing the way the movement is performed (e.g. position/orientation with respect to the environment, initial posture), and select among all the possible combinations the values that should be tested.

(2) Simulate the movement with an autonomous DHM for each selected combination of parameters values, and measure the associated performance indicators.

(3) Compute sensitivity measures for the performance indicators, based on their values in all the tested cases.

In step 1, the adjustable parameters and the numerical bounds within which these parameters are allowed to vary depend on the movement that is considered. As such, their choice is not addressed by the method described here. Instead the choice is left to the user. Appropriate numerical values to test for each of the selected parameters are determined according to the experimental design of the extended FAST method (Fourier amplitude sensitivity testing) (Saltelli, Tarantola, and Chan 1999). The FAST exploration method is a good compromise between the comprehensiveness of the space exploration and the number of trials.

In step 2, the DHM is animated with an LQP controller similar to the one used 
Table 1. Numerical values of the tasks weights used for dynamic replay.

\begin{tabular}{|c|c|c|}
\hline \multicolumn{2}{|c|}{ Task } & Weight \\
\hline \multicolumn{2}{|c|}{ balance } & 10 \\
\hline \multirow{7}{*}{ 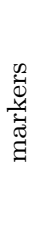 } & back & 1 \\
\hline & head & 2.5 \\
\hline & shoulder & 2.5 \\
\hline & elbow & 5 \\
\hline & hand & 20 \\
\hline & knee & 1 \\
\hline & ankle & 1 \\
\hline \multirow{9}{*}{$\begin{array}{l}0 \\
\Xi \\
\Xi \\
0 \\
0 \\
0 \\
0\end{array}$} & back & $10^{-1}$ \\
\hline & neck & $10^{-2}$ \\
\hline & scapula & $10^{-1}$ \\
\hline & shoulder & $10^{-2}$ \\
\hline & elbow & $10^{-3}$ \\
\hline & wrist & $10^{-3}$ \\
\hline & hip & $10^{-2}$ \\
\hline & knee & $10^{-2}$ \\
\hline & ankle & $10^{-2}$ \\
\hline \multicolumn{2}{|c|}{ torque minimization } & $10^{-8}$ \\
\hline
\end{tabular}

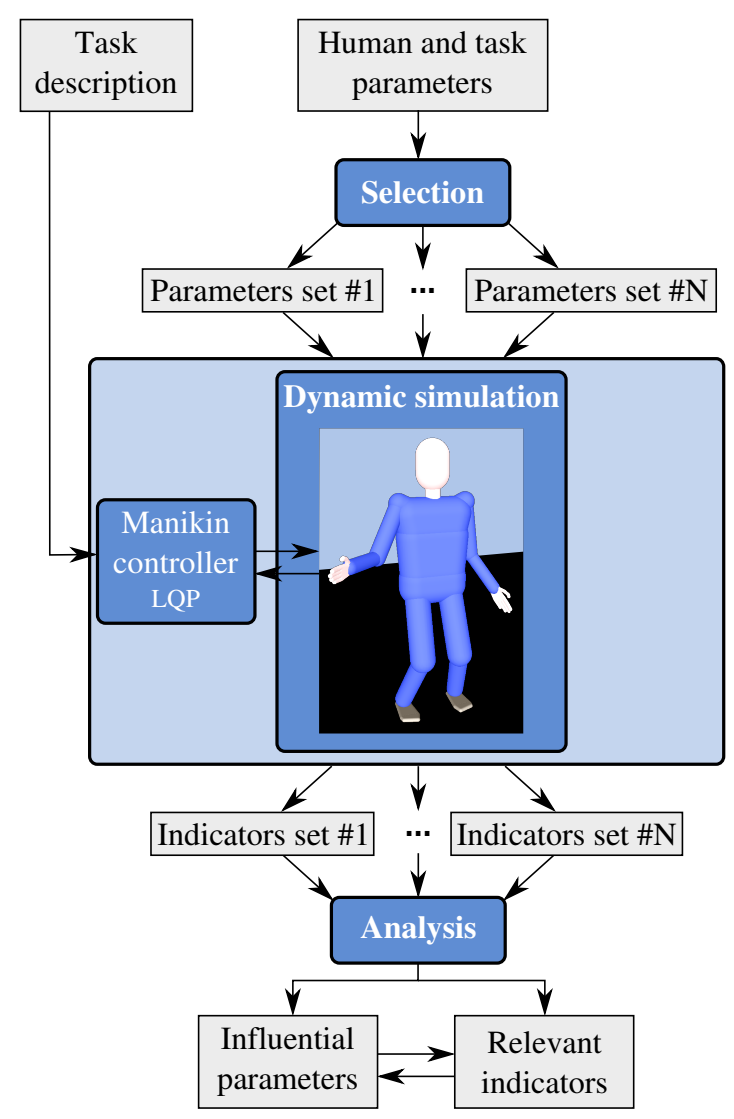

Figure 3. Flow chart of the method for analyzing the dependence between postural parameters of the task and resulting operational/biomechanical performances. 
for motion replay, but the markers tracking tasks are replaced by hands and/or feet operational acceleration tasks (depending on the goal of the movement). The reference trajectory - or at least the start and end points - must still be given as an input to the simulation.

A detailed assessment of biomechanical performances requires several indicators to account for the different demands (e.g. posture, effort, energy). The number of performance indicators should however be limited to facilitate the analysis, while sufficiently accounting for the overall performance. Hence, step 3 aims at identifying both the most relevant performance indicators and the most influential parameters (i.e. the parameters that have the strongest effects on the relevant indicators). In the context of performance improvement, the relevance of an indicator is not related to its value but to its variations when the movement is performed in different ways; if the value of an indicator remains unchanged whichever way the movement is performed, this indicator is not useful to compare different situations. Therefore indicators are ranked according to their variance, after they have been normalized to make the variances comparable. The number of indicators that should be kept is then chosen according to the Scree test (Jolliffe 2002).

Eventually, the influence of each parameter on the relevant performance indicators is estimated by computing Sobol indices which measure the percentage of variance of an indicator that is explained by the parameter (Hoeffding 1948; Sobol 1993). Sobol first order indices $S_{i}$ and total indices $S_{T_{i}}$ are used because they give information about the $i t h$ parameter $X_{i}$ independently from the influence of other parameters (Homma and Saltelli 1996). A high $S_{i}$ means that $X_{i}$ alone strongly affects the performance indicator, while a small $S_{T_{i}}$ means that $X_{i}$ has very little influence, even through interactions. This method allows to identify which parameters should mainly be tuned to improve the overall performance. It should be noted that Sobol indices represent relative contributions, i.e., they inform on the influence of a parameter compared to all parameters within the tested set.

\section{Experiment}

This section presents an application of the method for guiding performance enhancement presented in the previous sections. Human motions are recorded and replayed to evaluate the dynamic replay method. The sensitivity analysis is then applied to the considered movement, and the results are used to provide postural recommendations. The improved situation is compared to the original one to ensure that the proposed recommendations do enhance the performance. It should be noted that the application presented here is a proof of concept of the method proposed in this paper.

\subsection{Task description}

An industrial manual task requiring significant effort is used as a test case. The tasks consists in drilling six holes consecutively in a vertical slab of autoclaved aerated concrete (dimensions: $30 \times 60 \mathrm{~cm}$ ) with a portable electric drill. The locations of the holes are imposed and depicted on Fig. 4. The drill weighs $2.1 \mathrm{~kg}$. The average force needed to drill a hole in these conditions is around $40 \mathrm{~N}$ (measured with a force sensor embedded in the drill). The task duration is not constrained, but it takes about $1 \mathrm{~min}$ to perform the whole activity (take the drill, drill the six holes, put the drill down). Aside from the task correct execution (i.e. localization and depth of the holes), the 
main concern is the biomechanical performance: biomechanical demands should be minimized in order to decrease the risk of disease or injury.

\subsection{Motion capture set-up}

\subsubsection{Participants}

Five right-handed healthy students ( 3 males and 2 females) aged 25 to 30 years take part in the experiment (average size $1.72 \pm 0.1 \mathrm{~m}$; average body mass index $22.6 \pm$ $0.8 \mathrm{~kg} . \mathrm{m}^{-2}$ ). All participants gave informed consent before starting the experiment.

Each participant performs the task ten times, with a resting period between each trial. The drill is held with the right hand only. Participants choose their feet positions; they are allowed to move their feet between each trial but not within a trial.

\subsubsection{Instrumentation}

Participants' motions are recorded with a CodaMotion ${ }^{1}$ system at $100 \mathrm{~Hz}$. Participants are equipped with 25 markers spread all over their body (both legs, both arms, back and head). They stand on an AMTI force plate ${ }^{2}$ while performing the task to measure the GRF (for validation purpose only). A 6 axes ATI force sensor ${ }^{3}$ is embedded in the drill handle to measure the drilling forces (Fig. 4). The recorded data are filtered with a zero-phase $10 \mathrm{~Hz}$ low pass 4th order Butterworth filter. All recorded data are available upon request.

\subsubsection{Replay}

The recorded motions are replayed with a DHM using the dynamic replay method described in section 2.3. The drilling force measured with the force sensor is used as an input to the simulation, whereas the GRF measured with the force plate is used for validation purpose (in the simulation the GRF is automatically computed by the DHM controller).

Simulations are run in the XDE dynamic simulation framework developed by CEALIST (Merlhiot et al. 2012). The DHM consists of 21 rigid bodies linked together by 20 compound joints, for a total of 45 degrees of freedom (DoFs), plus 6 DoFs for the free-floating base. Each DoF is a revolute joint controlled by a single actuator. Given each participant's size and mass, the DHM is automatically scaled according to average anthropometric coefficients ${ }^{4}$. Each body segment can be further manually modified to match the participant's morphology when needed.

\subsection{Sensitivity analysis set-up}

\subsubsection{Postural parameters}

In manual tasks, postural parameters that can be adjusted are generally related to participants' position/orientation with respect to the environment. For the drilling

\footnotetext{
$1_{\text {www . codamotion. com }}$

2 http://www.amti.biz/

${ }^{3}$ http://www . ati-ia.com/products/ft/ft_models . aspx?id=Gamma

${ }^{4}$ segments lengths: http://www.openlab.psu.edu/tools/calculators/proportionalityConstant, segments masses: http://biomech.ftvs.cuni.cz/pbpk/kompendium/biomechanika/geometrie_hmotnost_vypocet_en
} 
task, nine parameters are defined and listed in Table 2, along with their user-defined limits. They include morphology-related parameters to check whether the formulated recommendations should depend on the the person's morphology or not. Note that the parameters used in this work are specific to the task addressed. The proposed analysis method is generic and can be applied to any movement, however the list of parameters and their bounds are movement-specific.

The R software sensitivity toolbox ${ }^{5}$ is used to select, within the user-defined numerical bounds, the parameters values that need to be tested for the extended FAST analysis. The sample size and set of frequencies are chosen based on the number of parameters, according to the recommendations of Saltelli et al. (Saltelli, Tarantola, and Chan 1999). They result in a total of 11601 simulations. One simulation takes approximately $2 \mathrm{~min}$ (real time: $75 \mathrm{~s}$ ) on one core of a $2.4 \mathrm{GHz}$ Intel R CoreTM i7 laptop. The simulations are parallelized on 8 cores.

\subsubsection{Simulations}

The drilling task is simulated in the XDE framework with the autonomous DHM. Only the right hand trajectory and force are explicitly specified. The reference hand trajectory and drilling force profile are estimated from the data recorded for the replay step. The DHM feet do not move during a simulation, except if balance cannot be maintained and the DHM falls. The correctness of the drilling task execution is ensured by checking the hand actual trajectory and force in each simulation.

\subsubsection{Performance indicators}

25 indicators are measured to assess the biomechanical performance. They are chosen to quantify as exhaustively as possible the effects of all kinds of physical demands, including dynamic phenomena (see Maurice et al. for a detailed description of the indicators (Maurice et al. 2014)). 20 indicators are local quantities which directly estimate joint demands: joint position, velocity, acceleration, power and torque for the right arm, left arm, back and legs respectively. 5 indicators are global quantities which represent the ability of a person to comfortably perform certain actions. The force ( resp. velocity) transmission ratio of the right hand estimates the capacity to produce force (resp. movement) in the drilling direction (Chiu 1987). The sum of the square distances between the center of pressure $(\mathrm{CoP})$ and the base of support boundaries (balance stability margin) (Xiang et al. 2010), and the time before the CoP reaches the base of support boundary (dynamic balance) estimate the balance quality. The kinetic energy of the whole body estimates human power consumption due to movement.

In order to make the variances comparable, the indicators must be scaled because they have non-homogeneous units and different orders of magnitude. Experimentally obtained reference values are used for the scaling (see (Maurice et al. 2014) for more details). To summarize each time-varying indicator in a single value, time-integral values over a whole simulation are used.

It should be noted that these biomechanical indicators are independent from the method presented in section 2. The method can be used with any indicators of human performance that can be measured on a DHM.

\footnotetext{
${ }^{5}$ http://www.r-project.org
} 


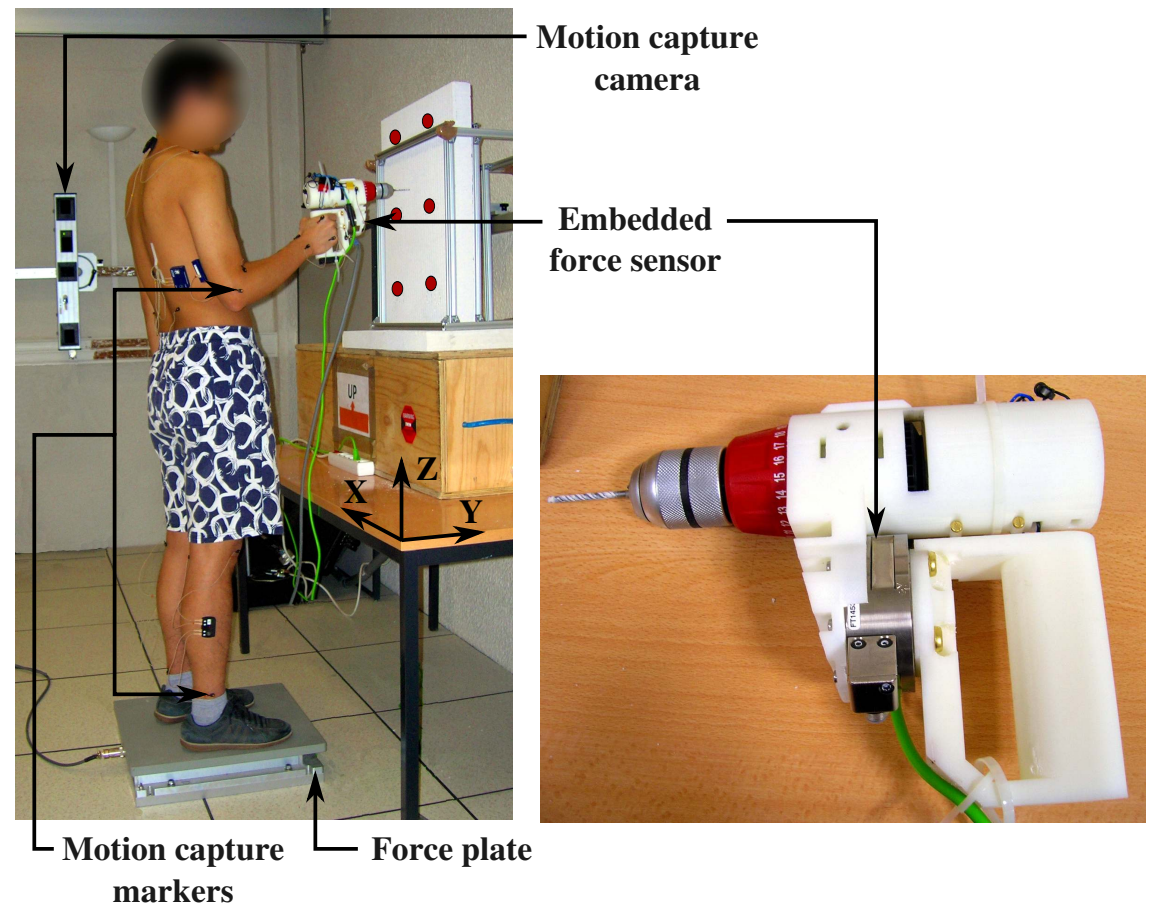

Figure 4. Motion and force capture instrumentation for the drilling task. A commercial drill has been modified to embed a force sensor. The red circles on the slab represent the drilling points.

Table 2. Parameters definition and limit values for the drilling task. The total horizontal distance between the pelvis and the stab center is equal to the value of the corresponding parameter plus the arm length of the DHM. The right foot is front when the inter-feet distance in the sagittal plane is positive. The influence of the person's morphology is taken into account both directly (with the DHM size and body mass index parameters) and indirectly since some parameters are partly calculated based on the DHM size (inter-feet distance in frontal plane, vertical distance between shoulder and stab center, horizontal distance between pelvis and stab center).

\begin{tabular}{lll}
\hline Parameter & Minimum & Maximum \\
\hline DHM size $(m)$ & 1.65 & 1.85 \\
DHM body mass index $\left(\mathrm{kg} \cdot \mathrm{m}^{-2}\right)$ & 21.0 & 27.0 \\
Preferred elbow flexion angle $\left(^{\circ}\right)$ & 10 & 135 \\
Inter-feet distance in frontal plane $(\%$ of hip width) & 100 & 200 \\
Inter-feet distance in sagittal plane $(m)$ & -0.25 & 0.25 \\
Orientation of drill handle w.r.t. vertical $\left(^{\circ}\right)$ & 0 & 90 \\
Pelvis horizontal orientation w.r.t. normal to stab $\left(^{\circ}\right)$ & -30 & 30 \\
Vertical distance between shoulder and stab center $(m)$ & -0.2 & 0.1 \\
Horizontal distance between pelvis and stab center $(m+$ DHM arm length) & -0.3 & 0.0
\end{tabular}




\section{Results}

This section presents the comparison between the recorded and replayed motions (Fig. 5), the output of the sensitivity analysis, and the comparison of the initial and improved situations.

\subsection{Dynamic replay validation}

\subsubsection{Motion}

The reliability of the replayed motion is assessed by comparing the 3D Cartesian positions of the experimental markers (recorded with the CodaMotion) with the simulated ones (points on the DHM body).

The RMS errors between the experimental and simulated markers positions are presented in Table 3 . The tracking error is smaller than $3 \mathrm{~cm}$ for all markers except the knee and right shoulder markers. The tracking error is smaller for the distal body parts, in accordance with the tasks weights distribution in the controller (higher weights for distal body parts). The tracking is better for the left arm than for the right arm because the left arm remains almost still. The right hand tracking error is nevertheless satisfactory (around $1 \mathrm{~cm}$ ), given that the overall length of the hand trajectory is about $1 \mathrm{~m}$. The results are consistent across participants.

\subsubsection{Force}

The reliability of the force data should be assessed by comparing the DHM joint torques computed with the controller with the human joint torques estimated from muscle forces. However, getting reliable human joint torques measurements is a practical issue. Conversely, the GRF is easily measured and provides an indirect estimation of the joint torques through the equation of motion (equality constraint in Eq. 1). The experimental GRF (measured with the force plate) and simulated GRF (computed with the DHM controller) are therefore compared.

The Pearson's linear correlation coefficient $r$ between the experimental and simulated GRF components is given in Table 4. A good correlation is observed for each GRF component (four components with $r \geq 0.90$, two components with $r \geq 0.70$ ). No significant permanent force/moment offset is observed (Fig. 6). $F_{Y}$ (direction of drilling) shows a better correlation than $F_{X}$ and $F_{Z}$ because the variations of $F_{Y}$ have a larger amplitude (Fig. 6). There are no significant differences across participants, except for the vertical force $F_{z}$. The disparity of the $F_{z}$ results might however be due to the lower precision of the force plate in this direction, because of the higher load.

\section{2. $\quad$ Sensitivity analysis}

Out of 25 biomechanical indicators, 6 are identified as relevant for the drilling task by the sensitivity analysis. These relevant indicators are given in Table 5 . Together they account for $80 \%$ of the total variance information, so little information is lost by not taking into account the other indicators. The presence of the upper-body torque and position indicators among the relevant indicators is consistent with the physical demands of the drilling task (exerting a significant force with the right hand while covering an extended area). The absence of any velocity and acceleration indicators is expected since the drilling task does not require fast motions. 


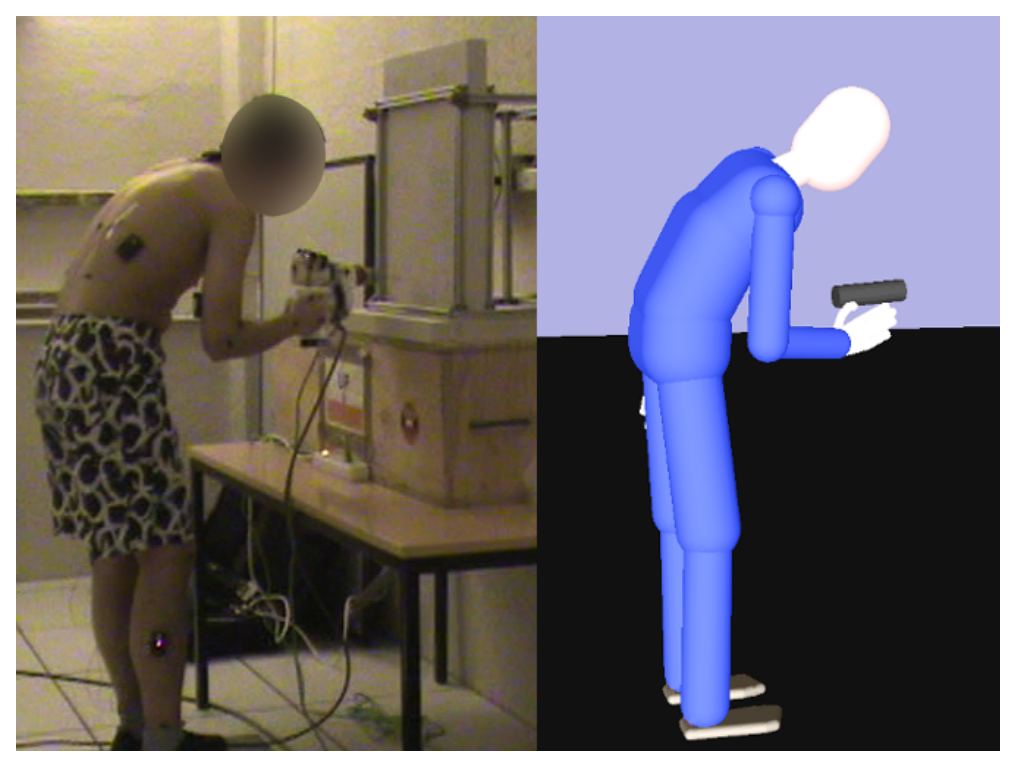

Figure 5. Motion capture (left) and dynamic replay with the LQP controller (right) of the drilling task. A video of the recorded and replayed motions is available in the supplementary material.

Table 3. Average RMS error between the experimental and simulated 3D markers positions across the 10 trials, for the 5 participants $\left(S_{i}\right.$ stands for subject $\left.i\right)$. Several markers are placed on each body/joint, but only the largest RMS error of all markers placed on a body/joint is given.

\begin{tabular}{lccccccc}
\hline \multicolumn{7}{c}{ Position } & RMS \\
& $S_{1}$ & $S_{2}$ & $S_{3}$ & $S_{4}$ & $S_{5}$ & Mean & SD \\
\hline Ankle & 1.5 & 1.0 & 0.9 & 1.1 & 1.3 & $\mathbf{1 . 2}$ & 0.2 \\
Knee & 4.6 & 4.9 & 3.7 & 3.8 & 4.8 & $\mathbf{4 . 4}$ & 0.5 \\
Back & 2.9 & 3.2 & 1.7 & 2.5 & 3.1 & $\mathbf{2 . 7}$ & 0.5 \\
Head & 1.6 & 1.6 & 0.6 & 1.5 & 1.0 & $\mathbf{1 . 3}$ & 0.4 \\
Right Shoulder & 7.8 & 6.9 & 2.0 & 6.8 & 7.0 & $\mathbf{6 . 1}$ & 2.1 \\
Left Shoulder & 3.9 & 2.7 & 1.9 & 2.2 & 3.5 & $\mathbf{2 . 8}$ & 0.8 \\
Right Elbow & 2.9 & 3.0 & 2.7 & 2.9 & 3.1 & $\mathbf{2 . 9}$ & 0.1 \\
Left Elbow & 0.8 & 0.5 & 0.3 & 0.8 & 0.7 & $\mathbf{0 . 6}$ & 0.2 \\
Right Wrist/Hand & 0.8 & 1.0 & 1.5 & 0.7 & 1.3 & $\mathbf{1 . 1}$ & 0.3 \\
Left Wrist/Hand & 0.5 & 0.4 & 0.2 & 0.3 & 0.2 & $\mathbf{0 . 3}$ & 0.1 \\
\hline
\end{tabular}

Table 4. Average Pearson's correlation coefficient between the simulated and experimental GRF components across the 10 trials, for the 5 participants $\left(S_{i}\right.$ stands for subject $i$ ). $X$ is the sagittal axis, $Y$ the frontal axis (drilling direction), and $Z$ the vertical axis.

\begin{tabular}{lllllll}
\hline & \multicolumn{3}{l}{ Force } & \multicolumn{5}{l}{ Moment } \\
\hline & $\mathbf{F}_{\mathbf{X}}$ & $\mathbf{F}_{\mathbf{Y}}$ & $\mathbf{F}_{\mathbf{Z}}$ & $\mathbf{M}_{\mathbf{X}}$ & $\mathbf{M}_{\mathbf{Y}}$ & $\mathbf{M}_{\mathbf{Z}}$ \\
\hline$S_{1}$ & 0.82 & 0.98 & 0.70 & 0.78 & 0.98 & 0.96 \\
$S_{2}$ & 0.82 & 0.98 & 0.62 & 0.95 & 0.98 & 0.95 \\
$S_{3}$ & 0.62 & 0.98 & 0.57 & 0.96 & 0.98 & 0.96 \\
$S_{4}$ & 0.78 & 0.98 & 0.91 & 0.96 & 0.98 & 0.98 \\
$S_{5}$ & 0.77 & 0.98 & 0.82 & 0.96 & 0.98 & 0.97 \\
Average & $\mathbf{0 . 7 6}$ & $\mathbf{0 . 9 8}$ & $\mathbf{0 . 7 2}$ & $\mathbf{0 . 9 2}$ & $\mathbf{0 . 9 8}$ & $\mathbf{0 . 9 7}$ \\
SD & 0.07 & 0.00 & 0.13 & 0.07 & 0.00 & 0.01 \\
\hline
\end{tabular}



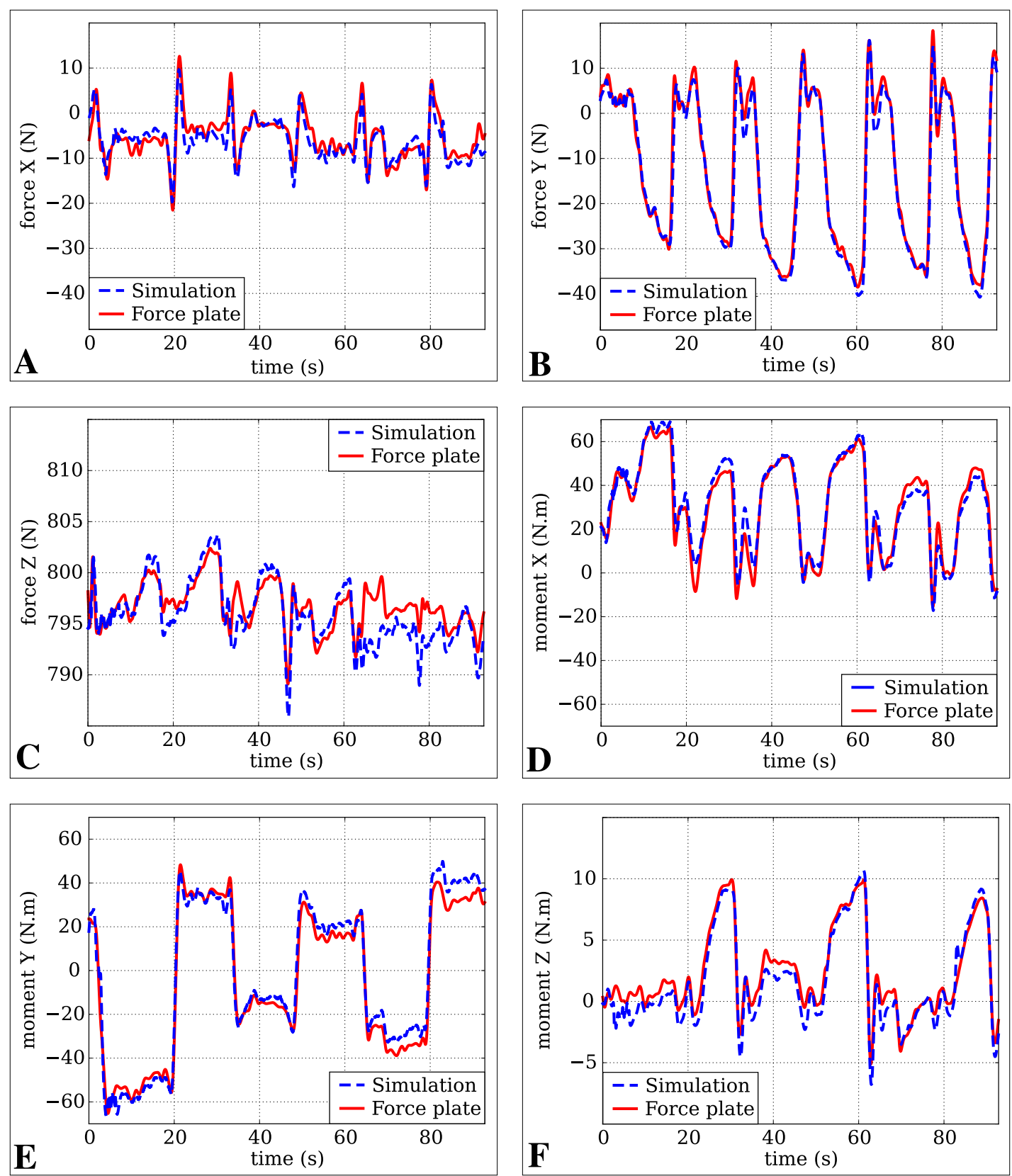

Figure 6. Time evolution of the experimental and simulated GRF components, for one trial of participant No.5. A: Force $F_{X}$. B: Force $F_{Y}$. C: Force $F_{Z}$. D: Moment $M_{X}$. E: Moment $M_{Y}$. F: Moment $M_{Z}$. The force and moment errors of this trial are representative of their average values across all participants and trials. The moments are given at the center of the feet. 
Table 5 also gives the value of Sobol indices for the 6 relevant indicators. Some parameter-indicator relations represented by these indices are expected and confirm the consistency of the proposed analysis (e.g. strong influence of the inter-feet frontal distance on the balance stability margin indicated by the high value of Sobol first order index). Other relations are less straightforward and could not easily be guessed without the sensitivity analysis, for instance the influence of the pelvis orientation on the right arm torque indicator. Overall, Sobol first order indices indicate that the pelvis orientation, the inter-feet sagittal distance, and the desired elbow flexion have a significant influence on several of the relevant indicators. These parameters should primarily be optimized to enhance the biomechanical performances. Conversely, low values of Sobol total indices indicate that the influences of the stab height and the pelvis-stab distance are small. The values of these parameters can be freely chosen by the person performing the activity. Surprisingly at first, the DHM morphological parameters are not identified as influent according to Sobol indices. This phenomenon can however be explained by the fact that other parameters are scaled based on the DHM size. Therefore, the set-up is adjusted depending on the DHM size, which likely reduces the influence of the morphological parameters on the biomechanical demands.

\subsection{Posture modification}

Sobol indices provide quantitative information about the magnitude of the parameters influence, but they do not inform on the detail of the indicators vs. parameters evolution. Such evolution can be estimated with a metamodel (Box and Draper 1987), but building a metamodel requires many more trials. As such, Sobol indices are not useful to find the parameters optimal values. However, trend curves can be obtained from the large number of trials performed for the sensitivity analysis, and used to identify well-performing parameters values. For each parameter, the optimal value is determined by considering only the associated relevant indicators. Recommendations for the drilling task are provided in Table 6. Pure morphological parameters (DHM size and body mass index) are excluded since they do not have a significant influence on the performance according to Table 5 .

The modified activity is compared to the initial one to validate the benefit resulting from the proposed recommendations. As a first validation, both the initial and the modified situations are evaluated with the autonomous DHM simulation (a complete validation would require the recording and replay of the movement performed by a human subject following the recommendations). The initial and recommended values of the parameters are displayed in Table 6 and the corresponding situations are illustrated in Fig. 7.

The values of the relevant indicators measured in both situations are presented in Table 7 . Out of the 6 relevant indicators, 5 are significantly improved by the proposed modifications, while the last one is only slightly worsened. Biomechanical demands associated with the right arm torque, legs position, right arm position, force transmission ratio and balance stability are reduced by $29,42,14,16$ and $35 \%$ respectively, whereas the back torque demand is increased by $9 \%$. Importantly, the significant reduction in biomechanical demand is achieved even though the range of variation of the adjustable parameter is limited. This result advocates for the use of the proposed method which can identify minor changes with a major impact.

The evolution of the shoulder flexion and rotation torques during the drilling movement are plotted in Fig. 8 to illustrate the reduced physical demands on more detailed 
Table 5. Sobol indices for the 6 biomechanical indicators identified as relevant for the drilling task. For each parameter and indicator, the upper value is the first order index, the lower value is the total index (Sobol indices can range from 0 to 1). The biomechanical indicators are presented in decreasing order of importance (decreasing variance) from left to right. The percentages below their names correspond to the percentage of the total variance they explain. FTR stands for force transmission ratio. Numbers are colored from blue (minimum) to red (maximum), to facilitate the reading.

\begin{tabular}{|c|c|c|c|c|c|c|c|}
\hline & & \multicolumn{6}{|c|}{ Relevant biomechanical indicators } \\
\hline & & $\begin{array}{l}\text { Right Arm } \\
\text { torque }\end{array}$ & $\begin{array}{l}\text { Legs } \\
\text { position }\end{array}$ & $\begin{array}{l}\text { Right Arm } \\
\text { position }\end{array}$ & $\begin{array}{l}\text { Back } \\
\text { torque }\end{array}$ & $\begin{array}{l}\text { FTR drilling } \\
\text { direction }\end{array}$ & $\begin{array}{l}\text { Balance stability } \\
\text { margin }\end{array}$ \\
\hline & & $23 \%$ & $20 \%$ & $15 \%$ & $9 \%$ & $7 \%$ & $6 \%$ \\
\hline \multirow{9}{*}{ 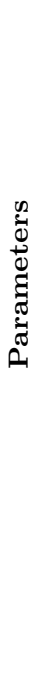 } & DHM size & $\begin{array}{l}10^{-3} \\
0.02\end{array}$ & $\begin{array}{l}0.01 \\
0.35\end{array}$ & $\begin{array}{l}10^{-3} \\
0.04\end{array}$ & $\begin{array}{l}0.02 \\
0.10\end{array}$ & $\begin{array}{l}0.09 \\
0.11\end{array}$ & $\begin{array}{l}0.03 \\
0.05\end{array}$ \\
\hline & DHM bmi & $\begin{array}{l}10^{-4} \\
0.03\end{array}$ & $\begin{array}{l}\mathbf{1 0}^{-\mathbf{3}} \\
0.31\end{array}$ & $\begin{array}{l}10^{-4} \\
0.10\end{array}$ & $\begin{array}{l}0.01 \\
0.10\end{array}$ & $\begin{array}{l}0.26 \\
0.30\end{array}$ & $\begin{array}{l}10^{-4} \\
0.03\end{array}$ \\
\hline & Elbow flexion & $\begin{array}{l}0.22 \\
0.26\end{array}$ & $\begin{array}{l}0.11 \\
\mathbf{0 . 6 7}\end{array}$ & $\begin{array}{l}0.03 \\
0.08\end{array}$ & $\begin{array}{l}10^{-3} \\
0.09\end{array}$ & $\begin{array}{l}0.02 \\
0.06\end{array}$ & $\begin{array}{l}10^{-3} \\
0.03\end{array}$ \\
\hline & $\begin{array}{l}\text { Inter-feet sagittal } \\
\text { distance }\end{array}$ & $\begin{array}{l}0.01 \\
0.03\end{array}$ & $\begin{array}{l}0.16 \\
0.66\end{array}$ & $\begin{array}{l}0.08 \\
0.15\end{array}$ & $\begin{array}{l}0.15 \\
0.30\end{array}$ & $\begin{array}{l}10^{-3} \\
0.02\end{array}$ & $\begin{array}{l}0.17 \\
0.23\end{array}$ \\
\hline & $\begin{array}{l}\text { Inter-feet frontal } \\
\text { distance }\end{array}$ & $\begin{array}{l}10^{-4} \\
0.02\end{array}$ & $\begin{array}{l}10^{-3} \\
0.31\end{array}$ & $\begin{array}{l}10^{-5} \\
10^{-3}\end{array}$ & $\begin{array}{l}0.01 \\
0.07\end{array}$ & $\begin{array}{l}10^{-4} \\
0.02\end{array}$ & $\begin{array}{l}0.53 \\
0.60\end{array}$ \\
\hline & $\begin{array}{l}\text { Drill } \\
\text { orientation }\end{array}$ & $\begin{array}{l}0.16 \\
0.26\end{array}$ & $\begin{array}{l}10^{-4} \\
0.32\end{array}$ & $\begin{array}{l}0.16 \\
0.24\end{array}$ & $\begin{array}{l}\mathbf{1 0}^{-\mathbf{3}} \\
0.12\end{array}$ & $\begin{array}{l}10^{-3} \\
0.05\end{array}$ & $\begin{array}{l}10^{-4} \\
0.02\end{array}$ \\
\hline & Pelvis orientation & $\begin{array}{l}0.32 \\
0.37\end{array}$ & $\begin{array}{l}\mathbf{0 . 0 2} \\
0.50\end{array}$ & $\begin{array}{l}0.45 \\
0.59\end{array}$ & $\begin{array}{l}0.43 \\
0.63\end{array}$ & $\begin{array}{l}0.45 \\
0.52\end{array}$ & $\begin{array}{l}0.10 \\
0.19\end{array}$ \\
\hline & Stab height & $\begin{array}{l}\mathbf{0 . 0 4} \\
0.09\end{array}$ & $\begin{array}{l}\mathbf{1 0}^{-4} \\
0.26\end{array}$ & $\begin{array}{l}0.05 \\
0.09\end{array}$ & $\begin{array}{l}\mathbf{0 . 0 6} \\
0.20\end{array}$ & $\begin{array}{l}0.04 \\
0.07\end{array}$ & $\begin{array}{l}10^{-4} \\
0.02\end{array}$ \\
\hline & Pelvis distance & $\begin{array}{l}0.09 \\
0.15\end{array}$ & $\begin{array}{l}\mathbf{0 . 0 1} \\
0.30\end{array}$ & $\begin{array}{l}0.06 \\
0.19\end{array}$ & $\begin{array}{l}0.01 \\
0.10\end{array}$ & $\begin{array}{l}0.04 \\
0.08\end{array}$ & $\begin{array}{l}10^{-4} \\
0.03\end{array}$ \\
\hline
\end{tabular}

Table 6. Initial and recommended parameters values for the drilling task. The parameters which have the largest influence on the performance are highlighted in bold. The values for the initial situation are measured on participant No.5.

\begin{tabular}{lll}
\hline Parameter & Initial & Recommended \\
\hline Preferred elbow flexion angle $\left(^{\circ}\right)$ & 100 & 135 \\
Inter-feet distance in frontal plane $(\%$ of hip width) & 120 & 200 \\
Inter-feet distance in sagittal plane $(m)$ & 0 & -0.1 \\
Orientation of drill handle w.r.t. vertical $\left(^{\circ}\right)$ & 0 & 0 \\
Pelvis horizontal orientation w.r.t. normal to stab $\left(^{\circ}\right)$ & 0 & -15 \\
Vertical distance between shoulder and stab center $(m)$ & 0 & -0.1 \\
Horizontal distance between pelvis and stab center $(m+$ DHM arm length) & 0 & 0.1 \\
\hline
\end{tabular}

Table 7. Values of the relevant biomechanical indicators measured in the initial and modified situations. FTR for Force Transmission Ratio. For each indicator, the value displayed is the percentage of the indicator reference value used for the scaling.

\begin{tabular}{cllllll}
\hline & $\begin{array}{l}\text { Right Arm } \\
\text { torque }\end{array}$ & $\begin{array}{l}\text { Legs } \\
\text { position }\end{array}$ & $\begin{array}{l}\text { Right Arm } \\
\text { position }\end{array}$ & $\begin{array}{l}\text { Back } \\
\text { torque }\end{array}$ & $\begin{array}{l}\text { FTR drilling } \\
\text { direction }\end{array}$ & $\begin{array}{l}\text { Balance stability } \\
\text { margin }\end{array}$ \\
\hline Init. & 138 & 69 & 126 & 76 & 128 & 106 \\
Modif. & $\mathbf{9 8}$ & 40 & $\mathbf{1 0 8}$ & $\mathbf{8 3}$ & $\mathbf{1 0 7}$ & $\mathbf{6 9}$ \\
\hline
\end{tabular}


biomechanical quantities. Both joint torques are reduced in the modified situation, and the DHM simulation allows a quantitative estimation of this reduction.

\section{Discussion}

The results presented in the previous section demonstrate the usefulness of the proposed method. On one hand, the dynamically replayed motion is very similar to the original one, and the simulated GRF is consistent with the experimental one. On the other hand, the situation modified according to the sensitivity analysis results exhibits enhanced performances, confirming the benefit of the postural modifications. Nevertheless, the application of the proposed method should be considered carefully because of some current limitations of the human model and control. Such limitations are discussed thereafter, along with leads on future research directions which may help lift those limitations.

\subsection{Accuracy of dynamic replay}

The tracking error of the markers 3D trajectories obtained with the dynamic replay method is similar to the tracking error reported for other replay methods including dynamic considerations (Table 3). Demircan et al. report a tracking error between 0 and $4 \mathrm{~cm}$ depending on markers, when replaying a throwing motion (Demircan et al. 2010). John and Dariush report an RMS tracking error of $4 \mathrm{~cm}$ for the worst marker, when tracking a set of 30 markers (John and Dariush 2014). This latter study however addresses seated motions where balance is less of an issue.

Though good, the replayed motion is nonetheless not exact (non-zero tracking error). In addition to soft tissue deformation and uncertainty on markers placement, the tracking errors are mainly due to differences between the human and DHM kinematics. For instance, the complexity of the human shoulder (De Sapio 2007) is not rendered in the DHM kinematics, hence the right shoulder tracking error. The balance task in the controller also alters the accuracy of the replayed motion. Due to differences between the human and DHM kinematics and the lack of decision skills of the DHM regarding how to recover balance, the ZMP preview control scheme in the balance task is tuned to be conservative. Most unstable situations are thus avoided as long as the original motion is not too unstable. However, the balance improvement is achieved at the cost of a modified motion, hence a less accurate replay. This partly explains the knee tracking error observed despite the small displacement of the knees during the drilling movement.

The kinematic differences between the human and the DHM can be minimized with more complex musculosketetal models, but they remain unavoidable and necessarily affect the quality of the replayed motion and forces (Hicks et al. 2015). These differences are, however, not specific to the dynamic replay method, but affect any kind of motion replay. Moreover, the replay method presented in this paper with a basic human model could also be used with more accurate musculosketetal models - though at a larger computational cost - to reduce model-induced errors. 

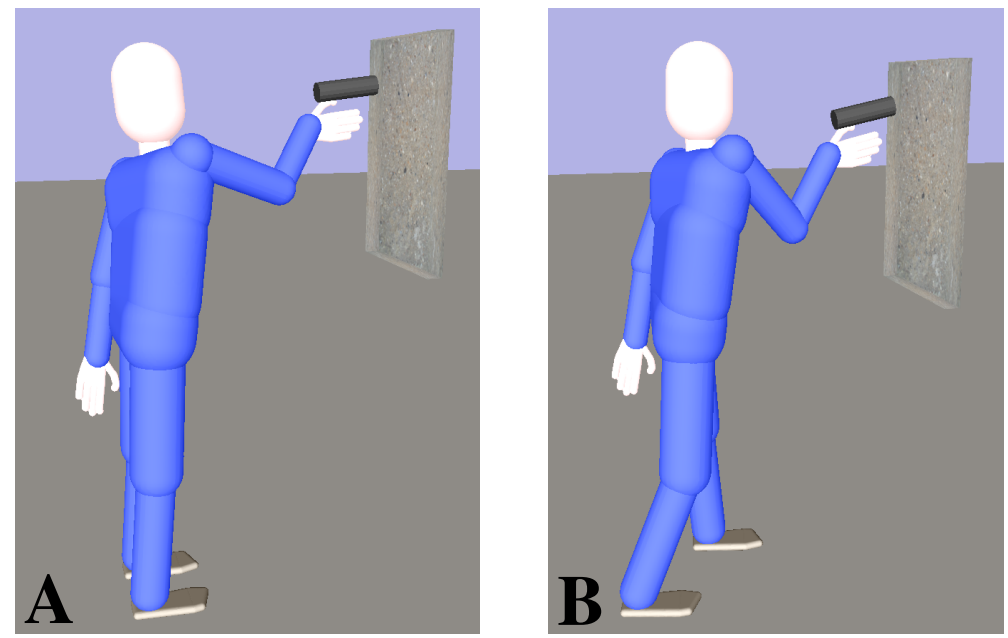

Figure 7. Snapshots of the initial (A) and modified (B) drilling movement simulated with the autonomous DHM.
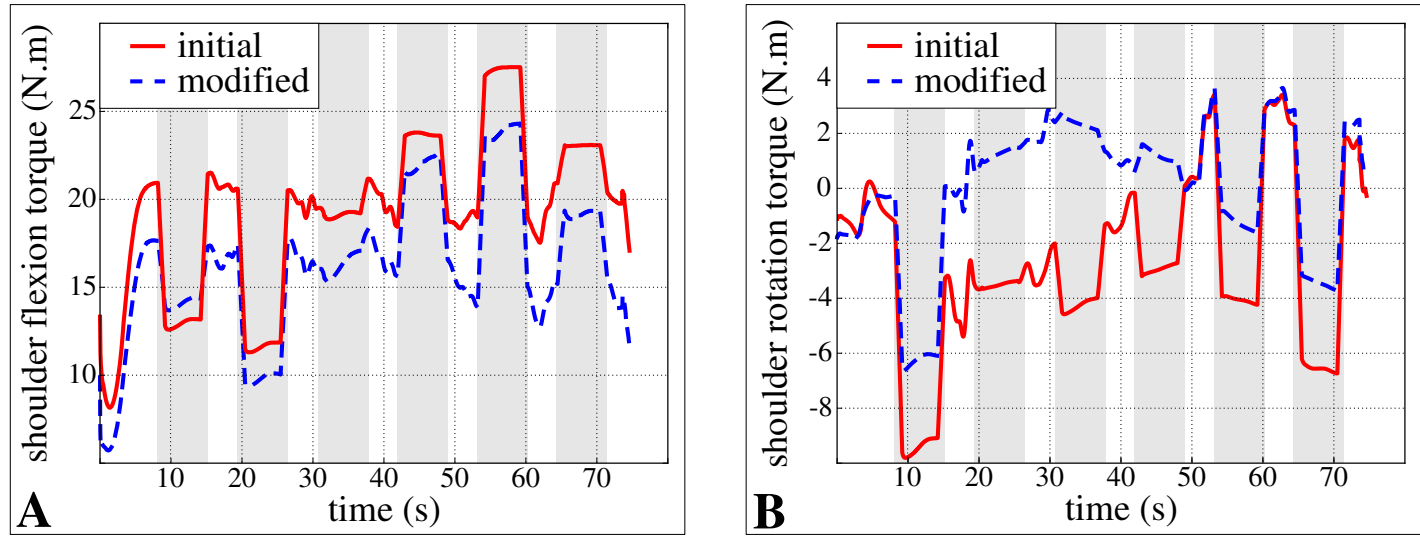

Figure 8. Time evolution of the shoulder flexion (A) and rotation (B) torques in the initial and modified situations. The grey areas correspond to the drilling periods. 


\subsection{Musculoskeletal model and motor control}

Unlike the muscular actuation of the human body, the actuation of the DHM is described at joint level only, with each DoF controlled by a single actuator. The biomechanical quantities measured with such a model are necessarily less detailed than what could be measured with a musculoskeletal model. Moreover, the DHM joint torques do not fully represent the physical effort exerted by a person. Due to the redundancy of human actuation, different combinations of muscle forces can result in a same joint torque. When simultaneously contracting antagonist muscles (co-contraction phenomenon), a person can generate forces which do not produce any net joint torque. These "internal forces" do not have any equivalent in the DHM and are not accounted for in the evaluation. Co-contraction of antagonist muscles aims at increasing joint impedance to withstand perturbations arising from limb dynamics or external loads (Gribble et al. 2003); though especially important in high accuracy motions, it is nevertheless present in all motions. Not taking co-contraction forces into account therefore leads to an under-estimation of the human effort.

Nevertheless, the motion replay method presented in this work is modular, in that it decouples the rigid body dynamics from the actuation dynamics. The output of the dynamic replay fully describes the evolution of the system dynamics in terms of state $\left[\mathbf{q}^{T}(t), \dot{\mathbf{q}}^{T}(t)\right]^{T}$, joint torques $\boldsymbol{\tau}(t)$ and applied external wrenches $\mathbf{w}_{\mathbf{c}}(t)$. Given this evolution, the use of an inverse musculoskeletal model (MM) could give access to the evolution of muscle activations $\mathbf{u}_{m}(t)=\mathbf{f}^{-1}\left(\mathbf{q}(t), \dot{\mathbf{q}}(t), \boldsymbol{\tau}(t), \mathbf{w}_{\mathbf{c}}(t), M M\right)$. Muscle-related performance indicators could thus be estimated. However the question of muscle recruitment in the $M M$ - especially regarding co-contraction - remains an open issue.

\subsection{Autonomous motion generation}

The sensitivity analysis being based on DHM simulations, the biomechanical reliability of the results depends on the realism of the autonomous DHM motion. Simulating highly realistic human motions requires a model of whole-body motor control which accounts for the redundancy of the human musculoskeletal system and for the slow dynamics of human muscle activation. The slow dynamics is particularly limiting as it requires to consider the motor control problem from an optimal control perspective rather than from a purely reactive perspective. The first consequence is a large increase in the computational cost, which is not compatible with running thousands of simulations in a reasonable amount of time. Moreover, solving this optimal control problem requires to understand the psychophysical principles that voluntary movements obey. Though many studies have been conducted to establish mathematical formulae of such principles (e.g. Fitt's law (Fitts 1954), minimum jerk principle (Flash and Hogan 1985), two-thirds power law (Viviani and Flash 1995)) and some have been successfully applied to DHM simulations (De Magistris et al. 2013), these formulations remain largely limited to reaching motions. Indeed, driving principles are not yet known for all kinds of whole-body motions, especially when significant external forces are at play. Transposed to a DHM, determining the underlying principles of human motion comes down to establishing which mathematical quantities are optimized when human-like motions are performed. Optimality criteria can be investigated through inverse optimization techniques - as proposed by Clever et al. (Clever, Hatz, and Mombaur 2014) for human locomotion or by Berret et al. (Berret et al. 2011) for reaching motions but they remain an open research problem. 
Nevertheless, the sensitivity analysis method in itself is independent from the DHM control. If an improved control law is available, it can be used to generate more realistic motions, while the analysis method remains the same.

\section{Conclusion}

This paper presents a framework to easily assess and enhance the performance of human postures and movements, based on dynamic DHM simulations. Existing situations are replayed from motion capture data with an LQP controller. This method guarantees the dynamic consistency of the replayed motion and forces, unlike what is currently achieved with most existing replay methods. The reliability of the biomechanical measurements taken on the DHM is thus increased, without requiring experimental GRF measurement. Then a sensitivity analysis of the movement is conducted with autonomous DHM simulations to identify the most influential parameters of the movement, and thereby provide recommendations for improvement. Because the DHM motion is automatically generated in this step, only little input data are needed to carry out the analysis. In particular, there is no need for human participants to perform multiple repetitions of the motion. The proposed method is applied to a drilling movement. Experiments carried out on 5 participants show that motions and forces are reliably replayed. The sensitivity analysis allows to highlight and rank some non trivial phenomena, which cannot be quantified a priori. Finally, the assessment of the modified situation shows significant improvement in performances compared to the initial situation, demonstrating the usefulness of the proposed method.

Futur work includes presenting the movement improvement tool to a human movement expert (e.g., ergonomist) to receive his/her feedback and compare the optimal movement obtained with the method proposed in this work to his/her own recommendations. Future research will also be directed towards coupling the developed tool with more detailed human models, such as musculo-skeletal models. Such a coupling will allow to measure quantities that more accurately represent the physiological demands during the movement. This will require investigating optimal coupling between the fast rigid-body simulation presented here and computationnally expensive musculoskeletal models, in order to keep the simulation time compatible with the execution of multiple simulation instances.

To conclude, though the application presented in this paper focuses on biomechanical performance, the method is more general and can be applied to other domains, such as rehabilitation.

\section{Funding}

This work was partially supported by the RTE company through the RTE/UPMC chair "Robotics Systems for field intervention in constrained environments", held by V. Padois. P. Maurice is supported in part by the European Union's Horizon 2020 research and innovation program under grant agreement No. 731540 (An.Dy). 


\section{References}

Berret, B., E. Chiovetto, F. Nori, and T. Pozzo. 2011. "Evidence for composite cost functions in arm movement planning: an inverse optimal control approach." PLoS Comput Biol 7 (10).

Box, G.E.P., and N.R. Draper. 1987. Empirical model-building and response surfaces. John Wiley \& Sons.

Chaffin, D.B., G.B.J. Andersson, and B.J. Martin. 2006. Occupational biomechanics. 4th ed. Wiley.

Chiu, SL. 1987. "Control of redundant manipulators for task compatibility." Proceedings of the IEEE International Conference on Robotics and Automation 4: 1718-1724.

Clever, D., K. Hatz, and K. Mombaur. 2014. "Studying Dynamical Principles of Human Locomotion using Inverse Optimal Control." Proceedings in Applied Mathematics and Mechanics $14(1): 801-802$.

Da Silva, M., Y. Abe, and J. Popović. 2008. "Simulation of Human Motion Data using ShortHorizon Model-Predictive Control." Computer Graphics Forum 27 (2): 371-380.

Damsgaard, M., J. Rasmussen, S. T. Christensen, E. Surma, and M. de Zee. 2006. "Analysis of musculoskeletal systems in the AnyBody Modeling System." Simulation Modelling Practice and Theory 14 (8): 1100-1111.

De Magistris, G., A. Micaelli, P. Evrard, C. Andriot, J. Savin, C. Gaudez, and J. Marsot. 2013. "Dynamic control of DHM for ergonomic assessments." International Journal of Industrial Ergonomics 43 (2): 170-180.

De Sapio, V. 2007. "Task-level strategies for constrained motion control and human motion synthesis." PhD diss., Stanford University.

Delp, S.L., F.C. Anderson, A.S. Arnold, P. Loan, A. Habib, C.T. John, E. Guendelman, and D.G. Thelen. 2007. "OpenSim: open-source software to create and analyze dynamic simulations of movement." IEEE Transactions on Biomedical Engineering 54 (11): 1940-1950.

Demircan, E. 2012. "Robotics-based Reconstruction and Synthesis of Human Motion." PhD diss., Stanford University.

Demircan, E., T. Besier, S. Menon, and O. Khatib. 2010. "Human motion reconstruction and synthesis of human skills." Advances in Robot Kinematics: Motion in Man and Machine 283-292.

Escande, A., N. Mansard, and P.B. Wieber. 2014. "Hierarchical quadratic programming: Fast online humanoid-robot motion generation." The International Journal of Robotics Research

Fitts, P.M. 1954. "The information capacity of the human motor system in controlling the amplitude of movement." Journal of experimental psychology 47 (6): 381.

Flash, T., and N. Hogan. 1985. "The coordination of arm movements: an experimentally confirmed mathematical model." The journal of Neuroscience 5 (7): 1688-1703.

Fortenbaugh, D., G.S. Fleisig, and J.R. Andrews. 2009. "Baseball pitching biomechanics in relation to injury risk and performance." Sports Health: A Multidisciplinary Approach 1 (4): $314-320$.

Gribble, P.L., L.I. Mullin, N. Cothros, and A. Mattar. 2003. "Role of cocontraction in arm movement accuracy." Journal of Neurophysiology 89 (5): 2396-2405.

Grochow, K., S.L. Martin, A. Hertzmann, and Z. Popović. 2004. "Style-based inverse kinematics." ACM Transactions on Graphics 23 (3): 522-531.

Hicks, J.L., T.K. Uchida, A. Seth, A. Rajagopal, and S.L. Delp. 2015. "Is My Model Good Enough? Best Practices for Verification and Validation of Musculoskeletal Models and Simulations of Movement." Journal of biomechanical engineering 137 (2).

Hoeffding, W. 1948. "A class of statistics with asymptotically normal distribution." The annals of mathematical statistics 293-325.

Homma, T., and A. Saltelli. 1996. "Importance measures in global sensitivity analysis of nonlinear models." Reliability Engineering 86 System Safety 52 (1): 1-17.

John, C., and B. Dariush. 2014. "Dynamically Consistent Human Movement Prediction for In- 
teractive Vehicle Occupant Package Design." Proceedings of the 3rd Digital Human Modeling Symposium .

Jolliffe, I. 2002. Principal component analysis. Wiley Online Library.

Kajita, S., F. Kanehiro, K. Kaneko, K. Fujiwara, K. Harada, K. Yokoi, and H. Hirukawa. 2003. "Biped walking pattern generation by using preview control of zero-moment point." Proceedings of the IEEE International Conference on Robotics and Automation 2: 16201626.

Khatib, O. 1987. "A unified approach for motion and force control of robot manipulators: The operational space formulation." IEEE Journal of Robotics and Automation 3 (1): 43-53.

Lee, J., and S.Y. Shin. 1999. "A hierarchical approach to interactive motion editing for humanlike figures." Proceedings of the 26th annual conference on Computer graphics and interactive techniques 39-48.

Maurice, P., V. Padois, Y. Measson, and P. Bidaud. 2017. "Human-oriented design of collaborative robots." International Journal of Industrial Ergonomics 57: 88-102.

Maurice, P., P. Schlehuber, V. Padois, Y. Measson, and P. Bidaud. 2014. "Automatic selection of ergonomie indicators for the design of collaborative robots: A virtual-human in the loop approach." 14th IEEE-RAS International Conference on Humanoid Robots 801-808.

Merlhiot, X., J. Le Garrec, G. Saupin, and C. Andriot. 2012. "The XDE mechanical kernel: Efficient and robust simulation of multibody dynamics with intermittent nonsmooth contacts." Proceedings of the 2nd Joint International Conference on Multibody System Dynamics .

Muico, U., Y. Lee, J. Popović, and Z. Popović. 2009. "Contact-aware nonlinear control of dynamic characters." ACM Transactions on Graphics 28 (3): 81.

Multon, F., R. Kulpa, L. Hoyet, and T. Komura. 2009. "Interactive animation of virtual humans based on motion capture data." Computer Animation and Virtual Worlds 20 (5-6): 491-500.

NRC. 2001. Musculoskeletal Disorders and the Workplace: Low Back and Upper Extremities. Institute of Medicine and National Research Council, National Academy Press.

Ott, C., D. Lee, and Y. Nakamura. 2008. "Motion capture based human motion recognition and imitation by direct marker control." Proceedings of the 8th IEEE-RAS International Conference on Humanoid Robots 399-405.

Robinson, Gemma, and Peter ODonoghue. 2008. "A movement classification for the investigation of agility demands and injury risk in sport." International Journal of Performance Analysis in Sport 8 (1): 127-144.

Salini, J., V. Padois, and P. Bidaud. 2011. "Synthesis of complex humanoid whole-body behavior: a focus on sequencing and tasks transitions." Proceedings of the IEEE International Conference on Robotics and Automation 1283-1290.

Saltelli, A., K. Chan, and E.M. Scott. 2000. Sensitivity analysis. Wiley.

Saltelli, A., S. Tarantola, and K.P.S. Chan. 1999. "A quantitative model-independent method for global sensitivity analysis of model output." Technometrics 41 (1): 39-56.

Schneider, E., and X. Irastorza. 2010. OSH in figures: Work-related musculoskeletal disorders in the EU - Facts and figures. European Agency for Safety and Health at Work.

Sentis, L., and O. Khatib. 2006. "A whole-body control framework for humanoids operating in human environments." Proceedings of the IEEE International Conference on Robotics and Automation 2641-2648.

Sobol, I.M. 1993. "Sensitivity estimates for non linear mathematical models." Mathematical Modelling and Computational Experiments 407-414.

Sturnieks, Daina L, Thor F Besier, Peter M Mills, Tim R Ackland, Ken F Maguire, Gwidon W Stachowiak, Pawel Podsiadlo, and David G Lloyd. 2008. "Knee joint biomechanics following arthroscopic partial meniscectomy." Journal of Orthopaedic Research 26 (8): 1075-1080.

Thelen, D.G., F.C. Anderson, and S.L. Delp. 2003. "Generating dynamic simulations of movement using computed muscle control." Journal of biomechanics 36 (3): 321-328.

Viviani, P., and T. Flash. 1995. "Minimum-jerk, two-thirds power law, and isochrony: converging approaches to movement planning." Journal of Experimental Psychology: Human Perception and Performance 21 (1): 32. 
Xiang, Y, J S Arora, S Rahmatalla, T Marler, R Bhatt, and K Abdel-Malek. 2010. "Human lifting simulation using a multi-objective optimization approach." Multibody System Dynamics 23 (4): 431-451. 